possible in the case of Lyme disease. Lyme disease parameters are not determined due to treatment interruption at the patient's request.

Conclusion A capite ad calcem approach is an essential part in dermatovenerology practice. It can help for doctor find not just seemingly the main reason for patient's complain but allow watch wider.

\section{P099 HIGH PREVALENCE OF CURABLE SEXUALLY TRANSMITTED INFECTIONS AMONG HIV-UNINFECTED WOMEN PLANNING FOR PREGNANCY IN KWAZULU- NATAL, SOUTH AFRICA}

${ }^{1}$ I Beesham*, ${ }^{1} \mathrm{~S}$ Bosman, ${ }^{1} \mathrm{M}$ Jaggernath, ${ }^{1} \mathrm{Y}$ Kriel, ${ }^{2} \mathrm{P}$ Chitneni, ${ }^{3} \mathrm{P}$ Smith, ${ }^{4} \mathrm{~K}$ Bennett, 1J Smit, ${ }^{3,5} \mathrm{~L}$ Matthews. ${ }^{1} \mathrm{MRU}$ (MatCH Research Unit), Faculty of Health Sciences, University of Witwatersrand, Durban, South Africa; ${ }^{2}$ Brigham and Women's Hospital, Massachusetts General Hospital Center for Global Health, Boston, USA; ${ }^{3}$ University of Alabama at Birmingham, School of Medicine, Division of Infectious Diseases, Birmingham, USA; ${ }^{4}$ Bennett Statistical Consulting, Ballston Lake, USA; ${ }^{5}$ Division of Infectious Disease, Massachusetts General Hospital, Boston, USA

\subsection{6/sextrans-2021-sti.228}

Background HIV-exposed women in African settings planning to conceive are at heightened risk of acquiring sexually transmitted infections (STIs). STIs can increase HIV acquisition risks and cause maternal and child morbidity. Here, we describe the prevalence of curable STIs among HIV-uninfected women planning pregnancy with a partner who is living with HIV or of unknown serostatus in an HIV-endemic setting (eThekwini, KwaZulu-Natal (KZN)).

Methods This is a descriptive longitudinal sub-study nested within the Safer Conception for Women study. HIV-uninfected women, aged 18-35 years with personal or partner plans for pregnancy were enrolled from November 2019 in eThekwini, KZN. A questionnaire was administered, and women were examined for STIs, and tested for Chlamydia trachomatis (CT), Neisseria gonorrhoeae (NG), Trichomonas vaginalis (TV), and Mycoplasma genitalium (MG) via PCR of a provider-collected swab, and Treponema Pallidum (TP) via TPHA testing. Women with symptomatic and/or laboratory-confirmed STIs are treated and provided with partner notification letters. Repeat sample collection is scheduled after six months, and during pregnancy. Here, we report on prevalent curable STIs at baseline.

Results Among the 50 women enrolled, [median age: 24 (range: 19-33), HIV serostatus of partner: 47(94\%) unknown], $3(6 \%)$ reported STI symptoms, and 12 (24\%) had curable STI organisms detected on laboratory testing. CT was the most frequent pathogen $(6,12 \%)$, followed by TV $(5$, $10 \%)$, NG (2, 4\%) and MG (1, 2\%). Two women had >1 STI pathogen. No TP was detected. Baseline demographic and sexual behavioural characteristics did not differ between women with and without STIs.

Conclusion Our findings indicate a high prevalence of curable STIs, mostly asymptomatic, among HIV-uninfected women planning for a pregnancy in the next year. Given the impact of asymptomatic curable STIs on maternal and neonatal health, this population should be prioritized for objective STI testing.

\section{P100 \\ HIV TESTING AND ASSOCIATED FACTORS IN MEN WHO HAVE SEX WITH MEN BY URBANIZATION-LEVEL: A CROSS-SECTIONAL STUDY IN THE NETHERLANDS}

${ }^{1,2} \mathrm{~J}$ Leenen, ${ }^{1,2} \mathrm{~J}$ Wijers, ${ }^{3,4,5} \mathrm{C}$ den Daas, ${ }^{5,6} \mathrm{~J}$ de Wit, ${ }^{1,2,7} \mathrm{C}$ Hoebe, ${ }^{1,8} \mathrm{~N}$ Dukers-Muijrers ${ }^{*}$ ${ }^{1}$ Department of Sexual Health, Infectious Diseases and Environmental Health, South Limburg, Heerlen, The Neterlands; ${ }^{2}$ Public Health Service (GGD Zuid Limburg), Heerlen, The Netherlands; ${ }^{3}$ Department of Medical Microbiology, Care and Public Health Research Institute (CAPHRI), Maastricht University Medical Centre (MUMC+), Maastricht, The Netherlands; ${ }^{4}$ Aberdeen Health Psychology Group, Institute of Applied Health Sciences, University of Aberdeen, Aberdeen, UK; ${ }^{5}$ Center for Infectious Disease Control, National Institute for Public Health and the Environment, Bilthoven, The Netherlands; ${ }^{6}$ Department of Interdisciplinary Social Science, Faculty of Social and Behavioral Sciences, Utrecht University, Utrecht, The Netherlands; ${ }^{7}$ Centre for Social Research in Health, University of New South Wales, Sydney, Australia; ${ }^{8}$ Department of Social Medicine, Care and Public Health Research Institute (CAPHRI), Maastricht University Medical Centre (MUMC+), Maastricht, The Netherlands

\subsection{6/sextrans-2021-sti.229}

Background Testing is a key strategy towards elimination of HIV; preferably by regional targeted approaches. Regular HIV testing enables timely care and reduces HIV transmission. We assessed HIV-testing behaviour in men who have sex with men (MSM), and factors associated with never and not recent testing by urbanisation-level.

Methods The online 'Men \& Sexuality' survey was conducted in 3,815 MSM (mean age 36 years; the Netherlands, 2018). HIV-testing was defined as recent (<1year), not recent ( $\geq 1$ year), and never. Testing was evaluated as outcome (recent testing was reference) in multivariable multinominal regression analyses (calculating odds ratio's [ORs]). We evaluated associations with urbanization-level. We also evaluated associations with a range of sociodemographic/behavioral factors in analyses stratified by urbanization-level (high-urban [ $>2,500$ living addresses/km2] and non-high-urban $[\leq 2,500$ living addresses/ $\mathrm{km} 2]])$.

Results In high-urban areas, $68.4 \%$ of MSM, and in non-highurban areas, 54.9\%, recently tested; $11.8 \%$ in high-urban and $25.2 \%$ in non-high-urban areas, never tested (aOR: 1.89; $1.55-2.31, \mathrm{p}<0.001)$.

In high-urban areas: factors associated with never and not recent testing were younger age, fewer new sex partners, never notified for HIV, no condomless anal intercourse, and self-identification as bisexual (versus gay).

In non-high-urban areas: additionally associated were moderate/low perceived HIV severity, moderate/low HIV risk perception, and moderate/low share of MSM among friends (versus 'high'). In non-high-urban areas 27\% reported a high share of MSM among friends (high-urban: 38\%).

In non-high-urban areas, never tested MSM would prefer self-sampling/self-testing (high-urban: facility-based testing).

Conclusion The proportion of MSM who never tested was high $(25 \%)$ in non-high-urban areas in The Netherlands; twofold compared to high-urban areas. In non-high urban areas, MSM who have a more restricted MSM-defined social context, may be less likely to test, and may benefit from targeted outreach and test-barrier-reducing strategies, e.g. by self-testing/self-sampling testing. A regional approach, accounting for urbanization-related factors, to eliminate HIV is warranted. 